\title{
ON THE EXPONENTIAL ERGODICITY OF LÉVY-DRIVEN ORNSTEIN-UHLENBECK PROCESSES
}

\author{
JIAN WANG, ${ }^{*}$ Fujian Normal University
}

\begin{abstract}
Based on the explicit coupling property, the ergodicity and the exponential ergodicity of Lévy-driven Ornstein-Uhlenbeck processes are established.

Keywords: Lévy process; Ornstein-Uhlenbeck process; coupling property; exponential ergodicity

2010 Mathematics Subject Classification: Primary 60H10
\end{abstract}

Secondary 60J75; 60G51

\section{Introduction and main results}

Let $\left(X_{t}^{x}\right)_{t \geq 0}$ be a $d$-dimensional Ornstein-Uhlenbeck process, which is defined as the unique strong solution of the following stochastic differential equation:

$$
\mathrm{d} X_{t}=A X_{t} \mathrm{~d} t+\mathrm{d} Z_{t}, \quad X_{0}=x \in \mathbb{R}^{d} .
$$

Here $A$ is a real $d \times d$ matrix and $\left(Z_{t}\right)_{t \geq 0}$ is a Lévy process in $\mathbb{R}^{d}$. It is well known that $\left(X_{t}^{x}\right)_{t \geq 0}$ is a strong Markov process with the following form:

$$
X_{t}^{x}=\mathrm{e}^{t A} x+\int_{0}^{t} \mathrm{e}^{(t-s) A} \mathrm{~d} Z_{s} .
$$

The associated Markov semigroup acting on $B_{b}\left(\mathbb{R}^{d}\right)$, the class of all bounded measurable functions on $\mathbb{R}^{d}$, is given by

$$
P_{t} f(x):=\mathrm{E} f\left(X_{t}^{x}\right)=\int_{\mathbb{R}^{d}} f\left(\mathrm{e}^{t A} x+z\right) \pi_{t}(\mathrm{~d} z), \quad t \geq 0, x \in \mathbb{R}^{d}, f \in B_{b}\left(\mathbb{R}^{d}\right),
$$

where $\pi_{t}$ is the law of $\int_{0}^{t} \mathrm{e}^{(t-s) A} \mathrm{~d} Z_{s}$. Semigroups of the type (3) are generalized Mehler semigroups.

Let us recall that a Lévy process $Z=\left(Z_{t}\right)_{t \geq 0}$ with values in $\mathbb{R}^{d}$ is an $\mathbb{R}^{d}$-valued process defined on some stochastic basis $\left(\Omega, \mathcal{F},\left(\mathcal{F}_{t}\right)_{t \geq 0}, \mathrm{P}\right)$, continuous in probability, having stationary independent increments, càdlàg trajectories, and such that $Z_{0}=0, \mathrm{P}$-almost surely. It is well known that the characteristic exponent or the symbol $\Phi$ of $\left(Z_{t}\right)_{t \geq 0}$, defined by

$$
\mathrm{E}\left(\mathrm{e}^{\mathrm{i}\left\langle\xi, Z_{t}\right\rangle}\right)=\mathrm{e}^{-t \Phi(\xi)}, \quad \xi \in \mathbb{R}^{d},
$$

satisfies the following Lévy-Khintchine representation:

$$
\Phi(\xi)=\frac{1}{2}\langle Q \xi, \xi\rangle+\mathrm{i}\langle b, \xi\rangle+\int_{z \neq 0}\left(1-\mathrm{e}^{\mathrm{i}\langle\xi, z\rangle}+\mathrm{i}\langle\xi, z\rangle \mathbf{1}_{B(0,1)}(z)\right) v(\mathrm{~d} z) .
$$

Received 13 March 2012; revision received 16 May 2012.

* Postal address: School of Mathematics and Computer Science, Fujian Normal University, 350007, Fuzhou, P. R. China. Email address: jianwang@fjnu.edu.cn 
Here $Q \in \mathbb{R}^{d \times d}$ is a positive semidefinite matrix, $b \in \mathbb{R}^{d}$ is the drift vector, and $v$ is the Lévy measure, i.e. a $\sigma$-finite measure on $\mathbb{R}^{d} \backslash\{0\}$ such that $\int_{z \neq 0}\left(1 \wedge|z|^{2}\right) v(\mathrm{~d} z)<\infty$. Our main reference for Lévy processes is the monograph [9].

The starting point of our paper is the following result about the existence of invariant measures for Ornstein-Uhlenbeck processes, which was proven in [10, Theorem 4.1].

Theorem 1. Let $X=\left(X_{t}^{x}\right)_{t \geq 0}$ be a d-dimensional Ornstein-Uhlenbeck process determined by (1), where the real parts of all the eigenvalues of $A$ are negative. If the Lévy measure $v$ of Lévy process $Z$ satisfies

$$
\int_{\{|z| \geq 1\}} \log (1+|z|) v(\mathrm{~d} z)<\infty,
$$

then there exists an invariant measure $\mu$ such that, for any $A \in \mathcal{B}\left(\mathbb{R}^{d}\right)$,

$$
P_{t}(x, A) \rightarrow \mu(A) \quad \text { as } t \rightarrow \infty
$$

where $P_{t}(x, \mathrm{~d} z)$ is the transition kernel of the process $\left(X_{t}\right)_{t \geq 0}$.

We are very much interested in the ergodicity and the exponential ergodicity of OrnsteinUhlenbeck processes. The standard method yielding the ergodicity is to verify that the process is strong Feller and irreducible; see [4], [6], and [15]. The strong Feller property of OrnsteinUhlenbeck processes has been studied in [2], [5], [7], and [14]. In particular, according to [7, Theorem 1.1 and Proposition 2.1], if the Lévy measure $v$ of Lévy process $Z$ is infinite and has a density with respect to the Lebesgue measure, then the associated Ornstein-Uhlenbeck process $\left(X_{t}\right)_{t \geq 0}$ determined by (1) is strong Feller. We refer the reader to [8, Section 3] for some discussions about the irreducibility of Ornstein-Uhlenbeck processes. The novelty of this paper is the direct use of the coupling property in the proof of the ergodicity (and the exponential ergodicity) for Ornstein-Uhlenbeck processes. The coupling property of Ornstein-Uhlenbeck processes has been studied in [12] and [16]. As we will see in the last section, an obvious advantage of the coupling method lies in the succinctness of the proof, which yields both the ergodicity and the exponential ergodicity simply via the Lévy measure $v$.

Before stating our main results, we first introduce some necessary notation. Let $v$ be the Lévy measure of the Lévy process $\left(Z_{t}\right)_{t \geq 0}$; see (4). For every $\varepsilon>0$, define $v_{\varepsilon}$ on $\mathbb{R}^{d}$ as follows: for any $B \in \mathscr{B}\left(\mathbb{R}^{d}\right)$,

$$
v_{\varepsilon}(B)= \begin{cases}v(B) & \text { if } v\left(\mathbb{R}^{d}\right)<\infty, \\ v(B \backslash\{z:|z|<\varepsilon\}) & \text { if } v\left(\mathbb{R}^{d}\right)=\infty\end{cases}
$$

Recall that, for any two bounded measures $\mu_{1}$ and $\mu_{2}$ on $\left(\mathbb{R}^{d}, \mathcal{B}\left(\mathbb{R}^{d}\right)\right)$,

$$
\mu_{1} \wedge \mu_{2}:=\mu_{1}-\left(\mu_{1}-\mu_{2}\right)^{+},
$$

where $\left(\mu_{1}-\mu_{2}\right)^{ \pm}$refers to the Jordan-Hahn decomposition of the signed measure $\mu_{1}-\mu_{2}$. In particular, $\mu_{1} \wedge \mu_{2}=\mu_{2} \wedge \mu_{1}$, and

$$
\mu_{1} \wedge \mu_{2}\left(\mathbb{R}^{d}\right)=\frac{1}{2}\left[\mu_{1}\left(\mathbb{R}^{d}\right)+\mu_{2}\left(\mathbb{R}^{d}\right)-\left\|\mu_{1}-\mu_{2}\right\|_{\mathrm{var}}\right]
$$

where $\|\cdot\|_{\text {var }}$ stands for the total variation norm. Denote by $P_{t}(x, \cdot)$ the transition kernel of the process $X$. 
Theorem 2. Let $X=\left(X_{t}^{x}\right)_{t \geq 0}$ be a d-dimensional Ornstein-Uhlenbeck process determined by (1), where the real parts of all the eigenvalues of A are negative. Then the following two statements hold.

(i) If the Lévy measure $v$ of the Lévy process $Z$ satisfies $\int_{\{|z| \geq 1\}} \log (1+|z|) v(\mathrm{~d} z)<\infty$ and

$$
\inf _{|x| \leq \rho} v_{\varepsilon} \wedge\left(\delta_{x} * v_{\varepsilon}\right)\left(\mathbb{R}^{d}\right)>0
$$

for some constants $\varepsilon, \rho>0$, then the process $X$ is ergodic, i.e. there is a unique invariant measure $\mu$ such that, for any $x \in \mathbb{R}^{d}$,

$$
\lim _{t \rightarrow \infty}\left\|P_{t}(x, \cdot)-\mu\right\|_{\mathrm{var}}=0 .
$$

(ii) If the Lévy measure $v$ of the Lévy process $Z$ satisfies $\int_{\{|z| \geq 1\}}|z| v(\mathrm{~d} z)<\infty$ and

$$
\limsup _{\rho \rightarrow 0}\left[\frac{\sup _{|x| \leq \rho}\left\|v_{\varepsilon}-\left(\delta_{x} * v_{\varepsilon}\right)\right\|_{\text {var }}}{\rho}\right]<\infty
$$

for some constant $\varepsilon>0$, then the process $X$ is exponentially ergodic. More explicitly, there exist a unique invariant measure $\mu$ and two constants $\kappa, C>0$ such that, for any $x \in \mathbb{R}^{d}$ and $t>0$,

$$
\left\|P_{t}(x, \cdot)-\mu\right\|_{\mathrm{var}} \leq C(1+|x|) \exp (-\kappa t) .
$$

Remark 1. (i) Under (7) and for fixed $\varepsilon>0$, there exists $\rho>0$ such that

$$
\sup _{|x| \leq \rho}\left\|v_{\varepsilon}-\left(\delta_{x} * v_{\varepsilon}\right)\right\|_{\mathrm{var}} \leq v_{\varepsilon}\left(\mathbb{R}^{d}\right)
$$

and so

$$
\begin{aligned}
\inf _{|x| \leq \rho} v_{\varepsilon} \wedge\left(\delta_{x} * v_{\varepsilon}\right)\left(\mathbb{R}^{d}\right) & =\frac{1}{2} \inf _{|x| \leq \rho}\left[v_{\varepsilon}\left(\mathbb{R}^{d}\right)+\left(\delta_{x} * v_{\varepsilon}\right)\left(\mathbb{R}^{d}\right)-\left\|v_{\varepsilon}-\left(\delta_{x} * v_{\varepsilon}\right)\right\|_{\mathrm{var}}\right] \\
& =\frac{1}{2}\left[2 v_{\varepsilon}\left(\mathbb{R}^{d}\right)-\sup _{|x| \leq \rho}\left\|v_{\varepsilon}-\left(\delta_{x} * v_{\varepsilon}\right)\right\|_{\mathrm{var}}\right] \\
& \geq \frac{1}{2} v_{\varepsilon}\left(\mathbb{R}^{d}\right) \\
& >0 .
\end{aligned}
$$

This shows that (7) implies (6).

(ii) We mention that in many applications condition (6) is weak. For example, Schilling and Wang [12, Proposition 1.5] proved that (6) is satisfied when the Lévy measure $v$ of $\left(Z_{t}\right)_{t \geq 0}$ satisfies $v(\mathrm{~d} z) \geq \rho(z) \mathrm{d} z$ such that $\int_{\left\{\left|z-z_{0}\right| \leq \varepsilon\right\}} \mathrm{d} z / \rho(z)<\infty$ holds for some $z_{0} \in \mathbb{R}^{d}$ and some $\varepsilon>0$.

(iii) According to Theorem 6 below, the second assertion in Theorem 2 still holds if (7) is replaced by

$$
\limsup _{r \rightarrow 0} \frac{\sup _{|x| \leq r} \int_{\left\{\left|z-z_{0}\right| \leq \varepsilon\right\}}|\rho(z)-\rho(x+z)| \mathrm{d} z}{r}<\infty
$$

for some $z_{0} \in \mathbb{R}^{d}$ and some $\varepsilon>0$, where $\rho(z)$ is a Borel measurable function on $\mathbb{R}^{d} \backslash\{0\}$ such that $v(\mathrm{~d} z) \geq \rho(z) \mathrm{d} z$. 
The following result presents the exponential ergodicity for Ornstein-Uhlenbeck processes, under weaker integral conditions for the Lévy measure $v$ on the range $\left\{z \in \mathbb{R}^{d}:|z| \geq 1\right\}$.

Theorem 3. Let $X=\left(X_{t}^{x}\right)_{t \geq 0}$ be a d-dimensional Ornstein-Uhlenbeck process determined by (1), where the real parts of all the eigenvalues of $A$ are negative. If the Lévy measure $v$ satisfies

$$
\liminf _{|\xi| \rightarrow \infty} \frac{\int_{\{|z| \leq 1 /|\xi|\}}\langle z, \xi\rangle^{2} v(\mathrm{~d} z)}{\log (1+|\xi|)}>0
$$

and $\int_{\{|z| \geq 1\}}|z|^{\alpha} v(\mathrm{~d} z)<\infty$ for some constant $0<\alpha \leq 1$, then there exist a unique invariant measure $\mu$ and two constants $\kappa, C>0$ such that, for any $x \in \mathbb{R}^{d}$ and $t>0$,

$$
\left\|P_{t}(x, \cdot)-\mu\right\|_{\mathrm{var}} \leq C\left(1+|x|^{\alpha}\right) \exp (-\kappa t) .
$$

It is clear that Theorem 3 can be applied to the study of the exponential ergodicity for Ornstein-Uhlenbeck processes driven by $\alpha$-stable processes with $\alpha \in(0,2)$.

The remainder of this paper is organized as follows. Section 2 is devoted to the coupling property of Ornstein-Uhlenbeck processes, which is key to our main results. In Section 3 we will present the proofs of Theorems 2 and 3. Here, a general conclusion for the exponential ergodicity of Ornstein-Uhlenbeck processes is given (see Theorem 6), which improves the second assertion of Theorem 2.

\section{Coupling property}

In this section we are mainly concerned with the coupling property for the OrnsteinUhlenbeck process $X=\left(X_{t}^{x}\right)_{t \geq 0}$ given by (2). Recall that the process $X$ has successful couplings (or has the coupling property) if and only if, for any $x, y \in \mathbb{R}^{d}$,

$$
\lim _{t \rightarrow \infty}\left\|P_{t}(x, \cdot)-P_{t}(y, \cdot)\right\|_{\mathrm{var}}=0,
$$

where $P_{t}(x, \mathrm{~d} z)$ is the transition kernel of the process $X$ and $\|\cdot\|_{\text {var }}$ stands for the total variation norm. The coupling property has been intensively studied for Lévy processes on $\mathbb{R}^{d}$ and Ornstein-Uhlenbeck processes driven by Lévy processes on $\mathbb{R}^{d}$; see [3], [11], [12], [13], and [16]. Recently, by using the lower-bound conditions for the Lévy measure with respect to a nice reference probability measure, we have successfully obtained the coupling property for linear stochastic differential equations driven by noncylindrical Lévy processes on Banach spaces; see [18, Theorem 1.2].

Let $v$ be the Lévy measure corresponding to the Lévy process $\left(Z_{t}\right)_{t \geq 0}$; see (4). For every $\varepsilon>0$, define a finite measure $v_{\varepsilon}$ on $\mathbb{R}^{d}$ as in (5). For a $d \times d$ matrix $A$, we say that an eigenvalue $\lambda$ of $A$ is semisimple if the dimension of the corresponding eigenspace is equal to the algebraic multiplicity of $\lambda$ as a root of the characteristic polynomial of $A$. Note that, for symmetric matrices, all the eigenvalues are real and semisimple.

The following result generalizes [12, Theorem 1.1], and it presents the exponential rate for the coupling property of Ornstein-Uhlenbeck processes.

Theorem 4. Let $X=\left(X_{t}^{x}\right)_{t \geq 0}$ be the Ornstein-Uhlenbeck process given by (2), where the real parts of all the eigenvalues of $A$ are nonpositive and all the purely imaginary eigenvalues of $A$ are semisimple. If there exist two constants $\varepsilon, \rho>0$ such that

$$
\inf _{|x| \leq \rho} v_{\varepsilon} \wedge\left(\delta_{x} * v_{\varepsilon}\right)\left(\mathbb{R}^{d}\right)>0
$$


then there exists a constant $C_{1}>0$ such that, for all $x, y \in \mathbb{R}^{d}$ and $t>0$,

$$
\left\|P_{t}(x, \cdot)-P_{t}(y, \cdot)\right\|_{\mathrm{var}} \leq \frac{C_{1}(1+|x-y|)}{\sqrt{t}} .
$$

Furthermore, suppose that the real parts of all the eigenvalues of A are negative. If (9) is strengthened by

$$
\limsup _{\rho \rightarrow 0}\left[\frac{\sup _{|x| \leq \rho}\left\|v_{\varepsilon}-\left(\delta_{x} * v_{\varepsilon}\right)\right\|_{\mathrm{var}}}{\rho}\right]<\infty,
$$

then there exist two constants $\kappa, C_{2}>0$ such that, for all $x, y \in \mathbb{R}^{d}$ and $t>0$,

$$
\left\|P_{t}(x, \cdot)-P_{t}(y, \cdot)\right\|_{\mathrm{var}} \leq C_{2}(1+|x-y|) \exp (-\kappa t) .
$$

Remark 2. Condition (9) has been used to study the coupling property for Lévy processes on $\mathbb{R}^{d}$ (see [11, Theorem 1.1]) and Ornstein-Uhlenbeck processes driven by Lévy processes on $\mathbb{R}^{d}$ (see [12, Theorem 1.1]).

Proof of Theorem 4. The first required assertion has been proven in [12, Theorem 1.1], and so it suffices to prove the second assertion. For simplicity, define $T_{t}=\mathrm{e}^{t A}$ for $t \geq 0$. Since the real parts of all the eigenvalues of $A$ are negative,

$$
\left\|T_{t}\right\|_{\mathbb{R}^{d} \rightarrow \mathbb{R}^{d}}:=\sup _{x \in \mathbb{R}^{d},|x|=1}\left|T_{t} x\right| \leq c \mathrm{e}^{-\lambda t}
$$

for all $t>0$ and some constants $c, \lambda>0$; see, e.g. [10, Equation (2.8)]. For any $\varepsilon>0$, let $\left(Z_{t}^{\varepsilon}\right)_{t \geq 0}$ be a compound Poisson process on $\mathbb{R}^{d}$ with Lévy measure $v_{\varepsilon}$, which is well defined since $v_{\varepsilon}$ is a finite measure on $\mathbb{R}^{d}$. Then, $\left(Z_{t}^{\varepsilon}\right)_{t \geq 0}$ and $\left(Z_{t}-Z_{t}^{\varepsilon}\right)_{t \geq 0}$ are two independent Lévy processes on $\mathbb{R}^{d}$. It follows, in particular, that the random variables

$$
X_{t}^{\varepsilon, x}:=T_{t} x+\int_{0}^{t} T_{t-s} \mathrm{~d} Z_{s}^{\varepsilon} \quad \text { and } \quad \bar{X}_{t}^{\varepsilon}:=X_{t}^{x}-X_{t}^{\varepsilon, x}=\int_{0}^{t} T_{t-s} \mathrm{~d}\left(Z_{s}-Z_{s}^{\varepsilon}\right)
$$

are well defined on $\mathbb{R}^{d}$ and are independent for any $\varepsilon>0$ and $t \geq 0$.

Define

$$
X_{t}^{\varepsilon, 0}:=X_{t}^{\varepsilon, x}-T_{t} x=\int_{0}^{t} T_{t-s} \mathrm{~d} Z_{s}^{\varepsilon} .
$$

We will rewrite $X_{t}^{\varepsilon, 0}$ as follows. Construct a sequence $\left(\tau_{i}\right)_{i \geq 1}$ of independent and identically distributed random variables which are exponentially distributed with intensity $C_{\varepsilon}=v_{\varepsilon}\left(\mathbb{R}^{d}\right)$, and introduce a further sequence $\left(U_{i}\right)_{i \geq 1}$ of independent and identically distributed random variables on $\mathbb{R}^{d}$ with law $\bar{v}_{\varepsilon}=v_{\varepsilon} / C_{\varepsilon}$. We will assume that the random variables $\left(U_{i}\right)_{i \geq 1}$ are independent of the sequence $\left(\tau_{i}\right)_{i \geq 1}$. Then, according to [9, Theorem 4.3],

$$
Z_{t}^{\varepsilon}=\sum_{i=1}^{N_{t}} U_{i}
$$

for every $t \geq 0$, where $\left(N_{t}\right)_{t \geq 0}$ is a Poisson process of intensity $C_{\varepsilon}$, i.e.

$$
N_{t}=\sup \left\{k \geq 1: \sum_{i=1}^{k} \tau_{i} \leq t\right\}
$$


and here we set $\sum_{i \in \varnothing}=0$ by convention. Therefore, it is not difficult to check that

$$
X_{t}^{\varepsilon, 0}=0 \cdot \mathbf{1}_{\left\{\tau_{1}>t\right\}}+\sum_{k=1}^{\infty} \mathbf{1}_{\left\{\tau_{1}+\cdots+\tau_{k} \leq t<\tau_{1}+\cdots+\tau_{k+1}\right\}}\left(T_{t-\tau_{1}} U_{1}+\cdots+T_{t-\left(\tau_{1}+\cdots+\tau_{k}\right)} U_{k}\right) .
$$

Since

$$
\tau_{1}+\cdots+\tau_{N_{t}} \leq t<\tau_{1}+\cdots+\tau_{N_{t}+1}, \quad t \geq 0,
$$

it holds that, on the set $\left\{N_{t} \geq 1\right\}$,

$$
X_{t}^{\varepsilon, 0}=\sum_{k=1}^{N_{t}} T_{t-\sum_{i=1}^{k} \tau_{i}} U_{k} .
$$

Next, we will make use of the decomposition

$$
P_{t} g(x)=\mathrm{E}\left(g\left(X_{t}^{x}\right) \mathbf{1}_{\left\{N_{t}=0\right\}}\right)+P_{t}^{1} g(x), \quad g \in B_{b}\left(\mathbb{R}^{d}\right), t \geq 0, x \in \mathbb{R}^{d},
$$

where

$$
P_{t}^{1} g(x)=\mathrm{E}\left(g\left(X_{t}^{x}\right) \mathbf{1}_{\left\{N_{t} \geq 1\right\}}\right) .
$$

According to all the above statements, we know that, for any $g \in B_{b}\left(\mathbb{R}^{d}\right)$ and $x \in \mathbb{R}^{d}$,

$$
\begin{aligned}
P_{t}^{1} g(x) & =\mathrm{E}\left(\mathbf{1}_{\left\{N_{t} \geq 1\right\}} g\left(T_{t} x+\bar{X}_{t}^{\varepsilon}+X_{t}^{\varepsilon, 0}\right)\right) \\
& =\mathrm{E}\left(\mathbf{1}_{\left\{N_{t} \geq 1\right\}} g\left(T_{t} x+\bar{X}_{t}^{\varepsilon}+\sum_{k=1}^{N_{t}} T_{t-\sum_{i=1}^{k} \tau_{i}} U_{k}\right)\right) \\
& =\mathrm{E}\left(\mathbf{1}_{\left\{N_{t} \geq 1\right\}} g\left(T_{t} x+\bar{X}_{t}^{\varepsilon}+\sum_{k=1}^{N_{t}-1} T_{t-\sum_{i=1}^{k} \tau_{i}} U_{k}+T_{t-\sum_{i=1}^{N_{t}} \tau_{i}} U_{N_{t}}\right)\right) \\
& =\frac{1}{C_{\varepsilon}} \mathrm{E}\left(\mathbf{1}_{\left\{N_{t} \geq 1\right\}} \int_{\mathbb{R}^{d}} g\left(T_{t} x+\bar{X}_{t}^{\varepsilon}+\sum_{k=1}^{N_{t}-1} T_{t-\sum_{i=1}^{k} \tau_{i}} U_{k}+T_{t-\sum_{i=1}^{N_{t}} \tau_{i}} z\right) v_{\varepsilon}(\mathrm{d} z)\right) .
\end{aligned}
$$

Therefore, for any $x, y \in \mathbb{R}^{d}$,

$$
\begin{aligned}
& \mid P_{t}^{1} g(x)- P_{t}^{1} g(y) \mid \\
&=\frac{1}{C_{\varepsilon}}\left[\mathrm{E}\left(\mathbf{1}_{\left\{N_{t} \geq 1\right\}} \int_{\mathbb{R}^{d}} g\left(T_{t} x+\bar{X}_{t}^{\varepsilon}+\sum_{k=1}^{N_{t}-1} T_{t-\sum_{i=1}^{k} \tau_{i}} U_{k}+T_{t-\sum_{i=1}^{N_{t}} \tau_{i}} z\right) v_{\varepsilon}(\mathrm{d} z)\right)\right. \\
&\left.\quad-\mathrm{E}\left(\mathbf{1}_{\left\{N_{t} \geq 1\right\}} \int_{\mathbb{R}^{d}} g\left(T_{t} y+\bar{X}_{t}^{\varepsilon}+\sum_{k=1}^{N_{t}-1} T_{t-\sum_{i=1}^{k} \tau_{i}} U_{k}+T_{t-\sum_{i=1}^{N_{t}} \tau_{i}} z\right) v_{\varepsilon}(\mathrm{d} z)\right)\right] \\
&=\frac{1}{C_{\varepsilon}}\left[\mathrm { E } \left(\mathbf { 1 } _ { \{ N _ { t } \geq 1 \} } \left(\int _ { \mathbb { R } ^ { d } } g \left(T_{t} y+\bar{X}_{t}^{\varepsilon}+\sum_{k=1}^{N_{t}-1} T_{t-\sum_{i=1}^{k} \tau_{i}} U_{k}\right.\right.\right.\right. \\
&\left.\quad+T_{t-\sum_{i=1}^{N_{t}} \tau_{i}}\left(z+T_{\sum_{i=1}^{N_{t}} \tau_{i}}(x-y)\right)\right) v_{\varepsilon}(\mathrm{d} z) \\
&\left.\left.\left.\quad \int_{\mathbb{R}^{d}} g\left(T_{t} y+\bar{X}_{t}^{\varepsilon}+\sum_{k=1}^{N_{t}-1} T_{t-\sum_{i=1}^{k} \tau_{i}} U_{k}+T_{t-\sum_{i=1}^{N_{t}} \tau_{i}} z\right) v_{\varepsilon}(\mathrm{d} z)\right)\right)\right]
\end{aligned}
$$




$$
\begin{aligned}
& =\frac{1}{C_{\varepsilon}}\left[\mathrm { E } \left(\mathbf { 1 } _ { \{ N _ { t } \geq 1 \} } \left(\int_{\mathbb{R}^{d}} g\left(T_{t} y+\bar{X}_{t}^{\varepsilon}+\sum_{k=1}^{N_{t}-1} T_{t-\sum_{i=1}^{k} \tau_{i}} U_{k}+T_{t-\sum_{i=1}^{N_{t}} \tau_{i}} z\right)\right.\right.\right. \\
& \times v_{\varepsilon}\left(\mathrm{d} z-T_{\sum_{i=1}^{N_{t}} \tau_{i}}(x-y)\right) \\
& \left.\left.\left.-\int_{\mathbb{R}^{d}} g\left(T_{t} y+\bar{X}_{t}^{\varepsilon}+\sum_{k=1}^{N_{t}-1} T_{t-\sum_{i=1}^{k} \tau_{i}} U_{k}+T_{t-\sum_{i=1}^{N_{t}} \tau_{i}} z\right) v_{\varepsilon}(\mathrm{d} z)\right)\right)\right] \\
& \leq \frac{1-\mathrm{e}^{-C_{\varepsilon} t}}{C_{\varepsilon}}\|g\|_{\infty} \sup _{z \in \mathbb{R}^{d},|z| \leq c|x-y|}\left\|v_{\varepsilon}-\delta_{z} * v_{\varepsilon}\right\|_{\mathrm{var}} \\
& \leq\|g\|_{\infty} \frac{c \Gamma}{C_{\varepsilon}}|x-y|,
\end{aligned}
$$

where, in the first inequality we have used the facts that $\mathrm{P}\left(N_{t} \geq 1\right)=1-\mathrm{e}^{-C_{\varepsilon} t}$ for $t \geq 0$ and $\left\|T_{t}\right\|_{\mathbb{R}^{d} \rightarrow \mathbb{R}^{d}} \leq c$ for all $t \geq 0$; and in the last inequality we set

$$
\Gamma:=\sup _{\rho>0}\left[\frac{\inf _{|x| \leq \rho}\left\|v_{\varepsilon}-\left(\delta_{x} * v_{\varepsilon}\right)\right\|_{\mathrm{var}}}{\rho}\right],
$$

which is finite due to (10) and the fact that

$$
\sup _{|x| \leq \rho}\left\|v_{\varepsilon}-\left(\delta_{x} * v_{\varepsilon}\right)\right\|_{\mathrm{var}} \leq 2 C_{\varepsilon}, \quad \rho>0, \varepsilon>0 .
$$

On the other hand, we have

$$
\left|\mathrm{E}\left(g\left(X_{t}^{x}\right) \mathbf{1}_{\left\{N_{t}=0\right\}}\right)\right| \leq\|g\|_{\infty} \mathrm{e}^{-C_{\varepsilon} t}, \quad t \geq 0, g \in B_{b}\left(\mathbb{R}^{d}\right) .
$$

Combining all the estimates with (11), we obtain, for any $x, y \in \mathbb{R}^{d}$,

$$
\left|P_{t} g(x)-P_{t} g(y)\right| \leq 2\|g\|_{\infty} \mathrm{e}^{-C_{\varepsilon} t}+\frac{c \Gamma}{C_{\varepsilon}}\|g\|_{\infty}|x-y| .
$$

Having all the conclusions above at hand, we can follow the proof of [18, Theorem 1.3] to obtain the desired assertion. Since $\left\|T_{t}\right\|_{\mathbb{R}^{d} \rightarrow \mathbb{R}^{d}} \leq c \mathrm{e}^{-\lambda t}$ for all $t \geq 0$ and some constants $c, \lambda>0$, it follows from (2) that

$$
\left|X_{t}^{x}-X_{t}^{y}\right| \leq c \mathrm{e}^{-\lambda t}|x-y|, \quad x, y \in \mathbb{R}^{d}, t \geq 0 .
$$

Therefore, for any $0<s<t$ and $x, y \in \mathbb{R}^{d}$,

$$
\begin{aligned}
\left|P_{t} g(x)-P_{t} g(y)\right| & =\mathrm{E}\left|P_{s} g\left(X_{t-s}^{x}\right)-P_{s} g\left(X_{t-s}^{y}\right)\right| \\
& \leq 2\|g\|_{\infty} \mathrm{e}^{-C_{\varepsilon} s}+\frac{c \Gamma}{C_{\varepsilon}}\|g\|_{\infty}\left|X_{t-s}^{x}-X_{t-s}^{y}\right| \\
& \leq c_{1}\|g\|_{\infty}(1+|x-y|)\left(\mathrm{e}^{-C_{\varepsilon} s} \vee \mathrm{e}^{-\lambda(t-s)}\right)
\end{aligned}
$$

holds for some constant $c_{1}>0$. Setting $s=\lambda t /\left(C_{\varepsilon}+\lambda\right)$, we obtain the required assertion. 
According to the above proof, under condition (10), we can get the following gradient estimates for a modified version $\left(P_{t}^{1}\right)_{t \geq 0}$ of $\left(P_{t}\right)_{t \geq 0}$ (see (12)):

$$
\sup _{t \geq 0,\|g\|_{\infty}=1, x \in \mathbb{R}^{d}}\left|\nabla P_{t}^{1} g(x)\right|:=\sup _{t \geq 0,\|g\|_{\infty}=1, x \in \mathbb{R}^{d}} \limsup _{x \rightarrow y} \frac{\left|P_{t}^{1} g(y)-P_{t}^{1} g(x)\right|}{|y-x|}<\infty .
$$

Such estimates have been considered in [17, Theorem 3.1] and [18, Proposition 4.1] by using the formula for random shifts of the compound Poisson measures when the Lévy measure is required to have absolutely continuous lower bounds with respect to some nice reference measures, e.g. the Lebesgue measure on $\mathbb{R}^{d}$ or the Gaussian measure on the Wiener space. Here, our condition (10) is more general and the proof is more direct.

A close inspection of the proof of Theorem 4 gives the following result.

Corollary 1. Let $X=\left(X_{t}^{x}\right)_{t \geq 0}$ be the Ornstein-Uhlenbeck process given by (2), where the real parts of all the eigenvalues of $A$ are nonpositive and all the purely imaginary eigenvalues of $A$ are semisimple. If there exists a finite measure $\mu$ on $\mathbb{R}^{d}$ such that $v \geq \mu$ and

$$
\limsup _{\rho \rightarrow 0}\left[\frac{\sup _{|x| \leq \rho}\left\|\mu-\left(\delta_{x} * \mu\right)\right\|_{\mathrm{var}}}{\rho}\right]<\infty,
$$

then there exist two constants $\kappa, C>0$ such that, for all $x, y \in \mathbb{R}^{d}$ and $t>0$,

$$
\left\|P_{t}(x, \cdot)-P_{t}(y, \cdot)\right\|_{\mathrm{var}} \leq C(1+|x-y|) \exp (-\kappa t) .
$$

The following estimate $\left\|P_{t}(x, \cdot)-P_{t}(y, \cdot)\right\|_{\text {var }}$ for large values of $t$ is based on the characteristic exponent $\Phi(\xi)$ of the Lévy process $\left(Z_{t}\right)_{t \geq 0}$.

Theorem 5. Let $X=\left(X_{t}^{x}\right)_{t \geq 0}$ be a d-dimensional Ornstein-Uhlenbeck process determined by (1), where the real parts of all the eigenvalues of $A$ are negative. Assume that the Lévy measure $v$ of $Z$ satisfies $\int_{\{|z| \geq 1\}} \log (1+|z|) v(\mathrm{~d} z)<\infty$, and that the associated symbol $\Phi$ fulfills

$$
\liminf _{|\xi| \rightarrow \infty} \frac{\operatorname{Re} \Phi(\xi)}{\log (1+|\xi|)}>0
$$

Then there exist $t_{1}, C>0$ such that, for any $x, y \in \mathbb{R}^{d}$ and $t \geq t_{1}$,

$$
\left\|P_{t}(x, \cdot)-P_{t}(y, \cdot)\right\|_{\mathrm{var}} \leq C\left|\mathrm{e}^{t A}(x-y)\right| \varphi_{t}^{-1}(1),
$$

where, for $t, \rho>0$,

$$
\varphi_{t}(\rho):=\sup _{|\xi| \leq \rho} \int_{0}^{t} \operatorname{Re} \Phi\left(\mathrm{e}^{s A^{\top}} \xi\right) \mathrm{d} s
$$

and $A^{\top}$ denotes the transpose of the matrix $A$.

Proof. We first assume that the Lévy process $\left(Z_{t}\right)_{t \geq 0}$ is a pure jump process, i.e. $Q=0$ and $b=0$ in (4). According to [12, Theorem 1.7], it suffices to verify that

$$
\xi \mapsto \int_{0}^{\infty} \operatorname{Re} \Phi\left(\mathrm{e}^{s A^{\top}} \xi\right) \mathrm{d} s \quad \text { is locally bounded, }
$$

and there exists some $t_{0}>0$ such that

$$
\liminf _{|\xi| \rightarrow \infty} \frac{\int_{0}^{t_{0}} \operatorname{Re} \Phi\left(\mathrm{e}^{s A^{\top}} \xi\right) \mathrm{d} s}{\log (1+|\xi|)}>2 d+2 .
$$


First, since the driving Lévy process $\left(Z_{t}\right)_{t \geq 0}$ has no Gaussian part, according to [10, Theorem 4.1] (or [5, Proposition 2.2]) and the assumptions, the process $\left(X_{t}\right)_{t \geq 0}$ possesses an invariant measure $\mu$, which is an infinite divisible distribution with the characteristic exponent $\xi \mapsto \int_{0}^{\infty} \Phi\left(\mathrm{e}^{s A^{\top}} \xi\right) \mathrm{d} s$. In particular, the function

$$
\xi \mapsto \int_{0}^{\infty} \operatorname{Re} \Phi\left(\mathrm{e}^{s A^{\top}} \xi\right) \mathrm{d} s
$$

is well defined and locally bounded.

On the other hand, set

$$
c_{0}:=\liminf _{|\xi| \rightarrow \infty} \frac{\operatorname{Re} \Phi(\xi)}{\log (1+|\xi|)}>0
$$

Choosing $t_{0}>(2 d+2) / c_{0}$, we have

$$
\begin{aligned}
\lim _{|\xi| \rightarrow \infty} \frac{\int_{0}^{t_{0}} \operatorname{Re} \Phi\left(\mathrm{e}^{s A^{\top}} \xi\right) \mathrm{d} s}{\log (1+|\xi|)} & \geq \lim _{|\xi| \rightarrow \infty} \int_{0}^{t_{0}} \frac{\operatorname{Re} \Phi\left(\mathrm{e}^{s A^{\top}} \xi\right)}{\log \left(1+\left|\mathrm{e}^{s A^{\top}} \xi\right|\right)} \mathrm{d} s \frac{\inf _{0<s<t_{0}} \log \left(1+\left|\mathrm{e}^{s A^{\top}} \xi\right|\right)}{\log (1+|\xi|)} \\
& \geq \int_{0}^{t_{0}} \underline{\lim }_{|\xi| \rightarrow \infty} \frac{\operatorname{Re} \Phi\left(\mathrm{e}^{s A^{\top}} \xi\right)}{\log \left(1+\left|\mathrm{e}^{s A^{\top}} \xi\right|\right)} \mathrm{d} s \\
& >2 d+2,
\end{aligned}
$$

where the second inequality follows from the Fatou lemma and the fact that

$$
\lim _{|\xi| \rightarrow \infty} \frac{\inf _{0<s<t_{0}} \log \left(1+\left|\mathrm{e}^{s A^{\top}} \xi\right|\right)}{\log (1+|\xi|)}=1 .
$$

This proves the required assertion.

Next, we consider the general case. Let $\left(Y_{t}\right)_{t \geq 0}$ and $\left(Z_{t}\right)_{t \geq 0}$ be two independent Lévy processes, whose symbols are

$$
\Phi_{Y}(\xi)=\int_{z \neq 0}\left(1-\mathrm{e}^{\mathrm{i}\langle\xi, z\rangle}+\mathrm{i}\langle\xi, z\rangle \mathbf{1}_{B(0,1)}(z)\right) v(\mathrm{~d} z)
$$

and

$$
\Phi_{Z}(\xi)=\Phi(\xi)-\Phi_{Y}(\xi),
$$

respectively. Denote by $Q_{t}$ and $Q_{t}(x, \cdot)$ the semigroup and the transition function of the $d$-dimensional Ornstein-Uhlenbeck process driven by $\left(Y_{t}\right)_{t \geq 0}$. Similarly, $R_{t}$ and $R_{t}(x, \cdot)$ stand for the semigroup and the transition function of the $d$-dimensional Ornstein-Uhlenbeck process driven by $\left(Z_{t}\right)_{t \geq 0}$. Note that $Q_{t}(x, \cdot)$ is the transition kernel of an Ornstein-Uhlenbeck process driven by a pure jump Lévy process. Then

$$
\begin{aligned}
\left\|P_{t}(x, \cdot)-P_{t}(y, \cdot)\right\|_{\mathrm{var}} & =\sup _{\|f\|_{\infty} \leq 1}\left|P_{t} f(x)-P_{t} f(y)\right| \\
& =\sup _{\|f\|_{\infty} \leq 1}\left|Q_{t} R_{t} f(x)-Q_{t} R_{t} f(y)\right| \\
& \leq \sup _{\|g\|_{\infty} \leq 1}\left|Q_{t} g(x)-Q_{t} g(y)\right| \\
& =\left\|Q_{t}(x, \cdot)-Q_{t}(y, \cdot)\right\|_{\mathrm{var}} .
\end{aligned}
$$

This, along with the conclusion above for $Q(x, \mathrm{~d} z)$, completes the proof. 


\section{Proofs}

In this section we will apply the results of Section 2 to the study of the ergodicity and the exponential ergodicity for Ornstein-Uhlenbeck processes. It is well known that the coupling property along with the existence of a stationary measure can yield the ergodicity for the process, which motivates the proof of Theorem 2.

Proof of Theorem 2. As mentioned in Theorem 1, according to [10, Theorem 4.1] or [5, Proposition 2.2], the process $X$ has an invariant measure $\mu$. In particular, $\mu P_{t}(\cdot, \mathrm{d} z)=\mu(\mathrm{d} z)$ for any $t>0$, where $P_{t}(x, \mathrm{~d} z)$ is the transition kernel of the process $X$. On the other hand, by Theorem 4, (6) and the condition that the real parts of all the eigenvalues of $A$ are negative imply that there exists $C_{1}>0$ such that, for any $t>0$ and $x, y \in \mathbb{R}^{d}$,

$$
\left\|P_{t}(x, \cdot)-P_{t}(y, \cdot)\right\|_{\mathrm{var}} \leq \frac{C_{1}(1+|x-y|)}{\sqrt{t}} .
$$

That is, when $t \rightarrow \infty,\left\|P_{t}(x, \cdot)-P_{t}(y, \cdot)\right\|_{\text {var }}$ converges to 0 uniformly for all $x, y \in \mathbb{R}^{d}$ with bounded $|x-y|$. Note that, for any $x \in \mathbb{R}^{d}$ and $t>0$,

$$
\left\|P_{t}(x, \cdot)-\mu\right\|_{\mathrm{var}} \leq \int\left\|P_{t}(x, \cdot)-P_{t}(y, \cdot)\right\|_{\mathrm{var}} \mu(\mathrm{d} y) .
$$

This along with the statement above yields, for any $x \in \mathbb{R}^{d}$,

$$
\lim _{t \rightarrow \infty}\left\|P_{t}(x, \cdot)-\mu\right\|_{\mathrm{var}}=0 .
$$

We mention here that the proof above also yields the uniqueness of the invariant measure. Indeed, let $\mu_{1}$ and $\mu_{2}$ be invariant measures for the process $X$. Then

$$
\left\|\mu_{1}-\mu_{2}\right\|_{\mathrm{var}} \leq \int\left\|P_{t}(x, \cdot)-P_{t}(y, \cdot)\right\|_{\mathrm{var}} \mu_{1}(\mathrm{~d} x) \mu_{2}(\mathrm{~d} y)
$$

By combining this with the proof above and letting $t \rightarrow \infty$, we obtain $\mu_{1}=\mu_{2}$. This proves the first required assertion.

For the second assertion, by (7) and Theorem 4, we know that there exist $\theta, C_{2}>0$ such that, for any $t>0$ and $x, y \in \mathbb{R}^{d}$,

$$
\left\|P_{t}(x, \cdot)-P_{t}(y, \cdot)\right\|_{\mathrm{var}} \leq C_{2}(1+|x-y|) \mathrm{e}^{-\theta t} .
$$

We will claim that, under the assumption that $\int_{\{|z| \geq 1\}}|z| v(\mathrm{~d} z)<\infty$,

$$
\int|x| \mu(\mathrm{d} x)<\infty
$$

If this holds then, following the argument above, we have

$$
\begin{aligned}
\left\|P_{t}(x, \cdot)-\mu\right\|_{\mathrm{var}} & \leq \int\left\|P_{t}(x, \cdot)-P_{t}(y, \cdot)\right\|_{\mathrm{var}} \mu(\mathrm{d} y) \\
& \leq C_{2}(1+|x|) \mathrm{e}^{-\theta t} \int|y| \mu(\mathrm{d} y) \\
& \leq C_{3}(1+|x|) \mathrm{e}^{-\theta t}
\end{aligned}
$$

The required assertion follows. 
Next, we consider the proof of (13). For $t>0$, set $T_{t}=\mathrm{e}^{t A}$ and $Y_{t}=\int_{0}^{t} T_{t-s} \mathrm{~d} Z_{s}$. For simplicity, we assume that $Z$ is a Lévy process on $\mathbb{R}^{d}$ without the Gaussian part. Thus, according to the Lévy-Itô decomposition (see [9, Chapter 4]), there exist $b \in \mathbb{R}^{d}$ and a Poisson random measure $N$ on $[0, \infty) \times \mathbb{R}^{d} \backslash\{0\}$ with intensity measure $\mathrm{d} s \otimes v(\mathrm{~d} z)$ (where $\mathrm{d} s$ is the Lebesgue measure on $[0, \infty)$ ) such that

$$
\mathrm{d} Z_{s}=b \mathrm{~d} s+\int_{\{|z| \leq 1\}} z \tilde{N}(\mathrm{~d} s, \mathrm{~d} z)+\int_{\{|z|>1\}} z N(\mathrm{~d} s, \mathrm{~d} z),
$$

where $\tilde{N}(\mathrm{~d} s, \mathrm{~d} z)$ is the compensated Poisson measure on $[0, \infty) \times \mathbb{R}^{d} \backslash\{0\}$, i.e.

$$
\tilde{N}(\mathrm{~d} s, \mathrm{~d} z)=N(\mathrm{~d} s, \mathrm{~d} z)-\mathrm{d} s v(\mathrm{~d} z) .
$$

Hence, the integral $Y_{t}$ is defined by

$$
Y_{t}=\int_{0}^{t} T_{t-s} b \mathrm{~d} s+\int_{0}^{t} \int_{\{|z| \leq 1\}} T_{t-s} z \tilde{N}(\mathrm{~d} s, \mathrm{~d} z)+\int_{0}^{t} \int_{\{|z|>1\}} T_{t-s} z N(\mathrm{~d} s, \mathrm{~d} z) .
$$

Since the real parts of all the eigenvalues of $A$ are negative, $\left\|T_{t}\right\|_{\mathbb{R}^{d} \rightarrow \mathbb{R}^{d}} \leq c \mathrm{e}^{-\lambda t}$ for all $t>0$ and some constants $c, \lambda>0$; see, e.g. [10, Equation (2.8)]. Thus, for any $t>0$,

$$
\left|\int_{0}^{t} T_{t-s} b \mathrm{~d} s\right| \leq \int_{0}^{t}\left\|T_{t-s}\right\|_{\mathbb{R}^{d} \rightarrow \mathbb{R}^{d}}|b| \mathrm{d} s \leq c|b| \int_{0}^{t} \mathrm{e}^{-\lambda(t-s)} \mathrm{d} s \leq \frac{c}{\lambda}|b|,
$$

and, by using the Cauchy-Schwarz inequality and the fact that $\tilde{N}(\mathrm{~d} s, \mathrm{~d} z)$ is a square-integrable martingale measure, see [1, Chapter 4.2],

$$
\begin{aligned}
\mathrm{E}\left|\int_{0}^{t} \int_{\{|z| \leq 1\}} T_{t-s} z \tilde{N}(\mathrm{~d} s, \mathrm{~d} z)\right| & \leq\left(\mathrm{E}\left|\int_{0}^{t} \int_{\{|z| \leq 1\}} T_{t-s} z \tilde{N}(\mathrm{~d} s, \mathrm{~d} z)\right|^{2}\right)^{1 / 2} \\
& =\left(\int_{0}^{t} \int_{\{|z| \leq 1\}}\left|T_{t-s} z\right|^{2} v(\mathrm{~d} z) \mathrm{d} s\right)^{1 / 2} \\
& \leq\left(\int_{0}^{t}\left\|T_{t-s}\right\|_{\mathbb{R}^{d} \rightarrow \mathbb{R}^{d}}^{2} \mathrm{~d} s \int_{\{|z| \leq 1\}}|z|^{2} v(\mathrm{~d} z)\right)^{1 / 2} \\
& \leq \frac{c}{\lambda} \sqrt{\int_{\{|z| \leq 1\}}|z|^{2} v(\mathrm{~d} z)} .
\end{aligned}
$$

On the other hand, noting that the integral $\int_{0}^{t} \int_{\{|z|>1\}} T_{t-s} z N(\mathrm{~d} s, \mathrm{~d} z)$ is defined as the Riemann integral and $\int_{0}^{t} \int_{\{|z|>1\}} z N(\mathrm{~d} s, \mathrm{~d} z)$ is a compound Poisson process with intensity $v\left(\left\{z \in \mathbb{R}^{d}\right.\right.$, $|z|>1\})$, it follows from the argument in [1, Chapter 4.3.5] that $\int_{0}^{t} \int_{\{|z|>1\}} T_{t-s} z N(\mathrm{~d} s, \mathrm{~d} z)$ is an infinitely divisible random variable associated with a Lévy measure

$$
v_{t}(D):=\int_{0}^{t} \int T_{t-s}^{-1}\left(D \cap\left\{z \in \mathbb{R}^{d}:|z|>1\right\}\right) v(\mathrm{~d} z) \text { for } D \in \mathbb{R}^{d} \backslash\{0\} .
$$

Thus,

$$
\begin{aligned}
\int v_{t}(\mathrm{~d} z) & =\int_{0}^{t} \int_{\left\{\left|T_{t-s} z\right|>1\right\}} v(\mathrm{~d} z) \mathrm{d} s \\
& \leq \int_{0}^{t} \int_{\left\{|z|>c^{-1}\right\}} v(\mathrm{~d} z) \mathrm{d} s \\
& \leq t \int_{\left\{|z|>c^{-1}\right\}} v(\mathrm{~d} z) .
\end{aligned}
$$


That is, for any $t>0$, the Lévy measure $v_{t}$ is a finite measure. Thus, $\int_{0}^{t} \int_{\{|z|>1\}} T_{t-s} z N(\mathrm{~d} s, \mathrm{~d} z)$ can be regarded as the random variable $E_{t, 1}$ for some compound Poisson process $\left(E_{t, s}\right)_{s \geq 0}$ with bounded Lévy measure $v_{t}$. According to the explicit expression of the semigroup for the compound Poisson process, see the proof of [9, Theorem 25.3] or [11, Equation (2.1)], we obtain

$$
\mathrm{E}\left|\int_{0}^{t} \int_{\{|z|>1\}} T_{t-s} z N(\mathrm{~d} s, \mathrm{~d} z)\right| \leq \sum_{n=0}^{\infty} \frac{1}{n !} \int|z| v_{t}^{* n}(\mathrm{~d} z) \leq \sum_{n=0}^{\infty} \frac{1}{n !}\left(\int|z| v_{t}(\mathrm{~d} z)\right)^{n},
$$

where $v_{t}^{* n}$ is the $n$-fold convolution of $v_{t}$ and $v_{t}^{* 0}=\delta_{0}$. By using the fact that, for any $t>0$,

$$
\begin{aligned}
\int|z| \mathrm{d} v_{t}(\mathrm{~d} z) & =\int_{0}^{t} \int_{\left\{\left|T_{t-s} z\right|>1\right\}}\left|T_{t-s} z\right| v(\mathrm{~d} z) \mathrm{d} s \\
& \leq \int_{0}^{t}\left\|T_{t-s}\right\|_{\mathbb{R}^{d} \rightarrow \mathbb{R}^{d}} \mathrm{~d} s \int_{\left\{|z|>c^{-1}\right\}}|z| v(\mathrm{~d} z) \\
& \leq \frac{c}{\lambda} \int_{\left\{|z|>c^{-1}\right\}}|z| v(\mathrm{~d} z)
\end{aligned}
$$

we arrive at

$$
\mathrm{E}\left|\int_{0}^{t} \int_{\{|z|>1\}} T_{t-s} z N(\mathrm{~d} s, \mathrm{~d} z)\right| \leq \exp \left(\frac{c}{\lambda} \int_{\left\{|z|>c^{-1}\right\}}|z| v(\mathrm{~d} z)\right) .
$$

Combining with all the conclusions above, it follows that $\mathrm{E}\left|Y_{t}\right|$ is bounded uniformly for all $t>0$, i.e. $\sup _{t>0} \mathrm{E}\left|Y_{t}\right| \leq C_{0}$ for some absolutely constant $C_{0}$.

Furthermore, by (2), for any $m \geq 1$ and $t>0$,

$$
\left|X_{t}^{x}\right| \wedge m \leq\left|T_{t} x\right| \wedge m+\left|Y_{t}\right|
$$

and so

$$
\mathrm{E}\left(\left|X_{t}^{x}\right| \wedge m\right) \leq \mathrm{E}\left(\left|T_{t} x\right| \wedge m\right)+\mathrm{E}\left|Y_{t}\right| \leq\left(c \mathrm{e}^{-\lambda t}|x|\right) \wedge m+C_{0} .
$$

Integrating this inequality with $\mu(\mathrm{d} x)$, we obtain

$$
\mu(|x| \wedge m) \leq \mu\left[\left(c \mathrm{e}^{-\lambda t}|x|\right) \wedge m\right]+C_{0}, \quad t>0, m \geq 1 .
$$

Letting first $t \rightarrow \infty$ and then $m \rightarrow \infty$, we prove the required assertion (13). This completes the proof.

We note that the argument of Theorem 2 above yields the following result.

Corollary 2. Let $X=\left(X_{t}^{x}\right)_{t \geq 0}$ be a d-dimensional Ornstein-Uhlenbeck process determined by (1), where the real parts of all the eigenvalues of A are negative. If the Lévy measure $v$ of the Lévy process $Z$ satisfies (6) and $\int_{\{|z| \geq 1\}}|z| v(\mathrm{~d} z)<\infty$, then the process $X$ is ergodic in the sense of algebraic convergence, i.e. there exist a unique invariant measure $\mu$ and a positive constant $C$ such that, for any $x \in \mathbb{R}^{d}$ and $t>0$,

$$
\left\|P_{t}(x, \cdot)-\mu\right\|_{\mathrm{var}} \leq \frac{C(1+|x|)}{\sqrt{t}} .
$$

Furthermore, according to Corollary 1 and the proof of Theorem 2, we have the following conclusion for the exponential ergodicity of Ornstein-Uhlenbeck processes, which improves the second assertion of Theorem 2 . 
Theorem 6. Let $X=\left(X_{t}^{x}\right)_{t \geq 0}$ be a d-dimensional Ornstein-Uhlenbeck process determined by (1), where the real parts of all the eigenvalues of A are negative and the Lévy measure $v$ of the Lévy process $Z$ satisfies $\int_{\{|z| \geq 1\}}|z| v(\mathrm{~d} z)<\infty$. If there exists a finite measure $\mu$ on $\mathbb{R}^{d}$ such that $v \geq \mu$ and

$$
\limsup _{\rho \rightarrow 0}\left[\frac{\sup _{|x| \leq \rho}\left\|\mu-\left(\delta_{x} * \mu\right)\right\|_{\mathrm{var}}}{\rho}\right]<\infty,
$$

then there exist a unique invariant measure $\mu$ and two constants $\kappa, C>0$ such that, for any $x \in \mathbb{R}^{d}$ and $t>0$,

$$
\left\|P_{t}(x, \cdot)-\mu\right\|_{\mathrm{var}} \leq C(1+|x|) \exp (-\kappa t) .
$$

The proof of Theorem 3 is based on the following lemma.

Lemma 1. Let $X=\left(X_{t}^{x}\right)_{t \geq 0}$ be a d-dimensional Ornstein-Uhlenbeck process determined by (1), where the real parts of all the eigenvalues of A are negative, and the symbol for the Lévy process $Z$ satisfies

$$
\liminf _{|\xi| \rightarrow \infty} \frac{\operatorname{Re} \Phi(\xi)}{\log (1+|\xi|)}>0 .
$$

If there exists a constant $0<\alpha \leq 1$ such that the Lévy measure $v$ of the Lévy process $Z$ satisfies $\int_{\{|z| \geq 1\}}|z|^{\alpha} v(\mathrm{~d} z)<\infty$, then there exist a unique invariant measure $\mu$ and two constants $\kappa, C>0$ such that, for any $x \in \mathbb{R}^{d}$ and $t>0$,

$$
\left\|P_{t}(x, \cdot)-\mu\right\|_{\mathrm{var}} \leq C\left(1+|x|^{\alpha}\right) \exp (-\kappa t) .
$$

Proof. For $t, \rho>0$, define

$$
\varphi_{t}(\rho):=\sup _{|\xi| \leq \rho} \int_{0}^{t} \operatorname{Re} \Phi\left(\mathrm{e}^{s A^{\top}} \xi\right) \mathrm{d} s .
$$

According to Theorem 5 and the Markov property, there exists a constant $t_{1}>0$ such that, for any $t \geq t_{1}, s>0$, and $g \in B_{b}\left(\mathbb{R}^{d}\right)$,

$$
\begin{aligned}
\left|P_{t} g(x)-P_{t+s} g(x)\right| & =\left|\mathrm{E}\left(P_{t} g(x)-P_{t} g\left(X_{s}^{x}\right)\right)\right| \\
& \leq \mathrm{E}\left|P_{t} g(x)-P_{t} g\left(X_{s}^{x}\right)\right| \\
& =\mathrm{E}\left(\frac{\left|P_{t} g(x)-P_{t} g\left(X_{s}^{x}\right)\right|^{\alpha}}{\left|X_{s}^{x}-x\right|^{\alpha}}\left|P_{t} g(x)-P_{t} g\left(X_{s}^{x}\right)\right|^{1-\alpha}\left|X_{s}^{x}-x\right|^{\alpha}\right) \\
& \leq C_{t}^{\alpha}\|g\|_{\infty}^{\alpha}\left(2\|g\|_{\infty}\right)^{1-\alpha} \mathrm{E}\left(\left|X_{s}^{x}-x\right|^{\alpha}\right) \\
& =2^{1-\alpha} C_{t}^{\alpha}\|g\|_{\infty} \mathrm{E}\left(\left|T_{s} x-x+Y_{s}\right|^{\alpha}\right),
\end{aligned}
$$

where $T_{s} x=\mathrm{e}^{s A} x, Y_{s}=\int_{0}^{s} T_{s-u} \mathrm{~d} Z_{u}$, and

$$
C_{t}=\left\|T_{t}\right\|_{\mathbb{R}^{d} \rightarrow \mathbb{R}^{d}} \varphi_{t}^{-1}(1) .
$$

Since the real parts of all the eigenvalues of $A$ are negative, $\left\|T_{t}\right\|_{\mathbb{R}^{d} \rightarrow \mathbb{R}^{d}} \leq c \mathrm{e}^{-\lambda t}$ for all $t>0$ and some constants $c, \lambda>0$; see, e.g. [10, Equation (2.8)]. Therefore, for any $\alpha \in(0,1]$,

$$
\mathrm{E}\left(\left|T_{s} x-x+Y_{s}\right|^{\alpha}\right) \leq \mathrm{E}\left(\left(\left|T_{s} x-x\right|+\left|Y_{s}\right|\right)^{\alpha}\right) \leq\left|T_{s} x-x\right|^{\alpha}+\mathrm{E}\left|Y_{s}\right|^{\alpha}
$$

and

$$
\left|T_{s} x-x\right|^{\alpha} \leq\left|T_{s} x\right|^{\alpha}+|x|^{\alpha} \leq\left(1+c^{\alpha}\right)|x|^{\alpha},
$$


where we have used the fact that

$$
(a+b)^{\alpha} \leq a^{\alpha}+b^{\alpha}, \quad a, b \geq 0 .
$$

On the other hand, under the assumption that $\int_{\{|z| \geq 1\}}|z|^{\alpha} v(\mathrm{~d} z)<\infty$, one can follow the proof of (13) to verify that $\mathrm{E}\left|Y_{s}\right|^{\alpha}$ is uniformly bounded for all $s>0$, i.e. $\sup _{s>0} \mathrm{E}\left|Y_{s}\right|^{\alpha}<\infty$. Therefore, there exists a constant $C_{0}>0$ such that

$$
\sup _{s>0} \mathrm{E}\left(\left|T_{s} x-x+Y_{s}\right|^{\alpha}\right) \leq C_{0}\left(1+|x|^{\alpha}\right) .
$$

Combining this with all the conclusions above, we obtain

$$
\left|P_{t} g(x)-P_{t+s} g(x)\right| \leq 2^{1-\alpha} C_{0}\left(1+|x|^{\alpha}\right) C_{t}^{\alpha}\|g\|_{\infty} .
$$

That is,

$$
\left\|P_{t}(x, \cdot)-P_{t+s}(x, \cdot)\right\|_{\mathrm{var}} \leq 2^{1-\alpha} C_{0}\left(1+|x|^{\alpha}\right) C_{t}^{\alpha} .
$$

Letting $s \rightarrow \infty$ and noting that $\mu$ is the invariant measure of the process $X$,

$$
\left\|P_{t}(x, \cdot)-\mu\right\|_{\mathrm{var}} \leq 2^{1-\alpha} C_{0}\left(1+|x|^{\alpha}\right) C_{t}^{\alpha} .
$$

As mentioned above, $\left\|T_{t}\right\|_{\mathbb{R}^{d} \rightarrow \mathbb{R}^{d}} \leq c \mathrm{e}^{-\lambda t}$ for all $t>0$. On the other hand, since, for $t \geq t_{1}$, $\varphi_{t}(\rho) \geq \varphi_{t_{1}}(\rho)$ and $\lim _{\rho \rightarrow \infty} \varphi_{t_{1}}(\rho)=\infty$, it holds that $\varphi_{t}^{-1}(1) \leq \varphi_{t_{1}}^{-1}(1)<\infty$ for any $t \geq t_{1}$. Therefore, there exists $C>0$ such that, for any $t \geq t_{1}, C_{t}^{\alpha} \leq C \mathrm{e}^{-\lambda t}$, which along with the conclusion above yields the required assertion.

Proof of Theorem 3. According to Lemma 1, it is sufficient to verify that (8) implies (14). For any $\xi \in \mathbb{R}^{d}$ with large enough $|\xi|$,

$$
\begin{aligned}
\operatorname{Re} \Phi(\xi) & =\int_{z \neq 0}(1-\cos \langle\xi, z\rangle) v(\mathrm{~d} z) \\
& \geq \int_{0<|z| \leq / 1|\xi|}(1-\cos \langle\xi, z\rangle) v(\mathrm{~d} z) \\
& \geq \frac{\cos 1}{2} \int_{0<|z| \leq / 1|\xi|}\langle\xi, z\rangle^{2} v(\mathrm{~d} z),
\end{aligned}
$$

where in the second inequality we have used the inequality

$$
1-\cos r \geq \frac{\cos 1}{2} r^{2}, \quad|r| \leq 1
$$

This satisfies the desired assertion.

\section{Acknowledgements}

We would like to thank an anonymous referee whose careful reading helped to improve the presentation of this paper. Financial support through the National Natural Science Foundation of China (grant numbers 11126350 and 11201073) and the Programme of Excellent Young Talents and New Century Excellent Talents in Universities of Fujian (grant numbers JA10058, JA11051, and JA12053) is gratefully acknowledged. 


\section{References}

[1] Applebaum, D. (2005). Lévy Processes and Stochastic Calculus. Cambridge University Press.

[2] Bodnarchuk, S. V. And Kulik, O. M. (2009). Conditions for the existence and smoothness of the distribution density for an Ornstein-Uhlenbeck process with Lévy noise. Theoret. Prob. Math. Statist. 79, 23-38.

[3] Böttcher, B., Schilling, R. L. AND WANG, J. (2011). Constructions of coupling processes for Lévy processes. Stoch. Process. Appl. 121, 1201-1216.

[4] CERraI, S. (2001). Second Order PDE's in Finite and Infinite Dimension (Lecture Notes Math. 1762). Springer, Berlin.

[5] Masuda, H. (2004). On multidimensional Ornstein-Uhlenbeck processes driven by a general Lévy process. Bernoulli 10, 97-120.

[6] Meyn, S. P. And Tweedie, R. L. (1993). Markov Chains and Stochastic Stability. Springer, London.

[7] Priola, E. ANd ZabCZYK, J. (2009). Densities for Ornstein-Uhlenbeck processes with jumps. Bull. London Math. Soc. 41, 41-50.

[8] Priola, E. And ZabczyK, J. (2009). On linear evolution equations with cylindrical Lévy noise. Preprint. Available at http://arxiv.org/abs/0908.0356v1.

[9] Sato, K.-I. (1999). Lévy Processes and Infinitely Divisible Distributions. Cambridge University Press.

[10] Sato, K.-I. and Yamazato, M. (1984). Operator-self-decomposable distributions as limit distributions of processes of Ornstein-Uhlenbeck type. Stoch. Process. Appl. 17, 73-100.

[11] Schilling, R. L. And Wang, J. (2011). On the coupling property of Lévy processes. Ann. Inst. H. Poincaré Prob. Statist. 47, 1147-1159.

[12] Schilling, R. L. AND WANG, J. (2012). On the coupling property and the Liouville theorem for OrnsteinUhlenbeck processes. J. Evol. Equat. 12, 119-140.

[13] Schilling, R. L., SztonyK, P. And Wang, J. (2012). Coupling property and gradient estimates of Lévy processes via symbol. Bernoulli 18, 1128-1149.

[14] Simon, T. (2011). On the absolute continuity of multidimensional Ornstein-Uhlenbeck processes. Prob. Theory Relat. Fields 151, 173-190.

[15] Stettner, Ł. (1994). Remarks on ergodic conditions for Markov processes on Polish spaces. Bull. Polish Acad. Sci. Math. 42, 103-114.

[16] WANG, F.-Y. (2011). Coupling for Ornstein-Uhlenbeck jump processes with jumps. Bernoulli 17, 1136-1158.

[17] Wang, F.-Y. (2011). Gradient estimate for Ornstein-Uhlenbeck jump processes. Stoch. Process. Appl. 121, 466-478.

[18] Wang, F.-Y. AND WANG, J. (2012). Coupling and strong Feller for jump processes on Banach spaces. Preprint. Available at http://arxiv.org/abs/1111.3795v2. 